\title{
Antiglycation Activities and Common Mechanisms Mediating Vasculoprotective Effect of Quercetin and Chrysin in Metabolic Syndrome
}

\author{
Osama A. A. Ahmed $\mathbb{D}^{1,2}$ Ahmad S. Azhar ${ }^{10},{ }^{3}$ Mayada M. Tarkhan, ${ }^{4}$ \\ Khadijah S. Balamash $\left(\mathbb{1},{ }^{4}\right.$ and Hany M. El-Bassossy ${ }^{5}$ \\ ${ }^{1}$ Department of Pharmaceutics, Faculty of Pharmacy, King Abdulaziz University, Jeddah, Saudi Arabia \\ ${ }^{2}$ Department of Pharmaceutics and Industrial Pharmacy, Faculty of Pharmacy, Minia University, Minia, Egypt \\ ${ }^{3}$ Pediatric Cardiac Center of Excellence, Faculty of Medicine, King Abdulaziz University, Jeddah, Saudi Arabia \\ ${ }^{4}$ Department of Biochemistry, Faculty of Science, King Abdulaziz University, Jeddah, Saudi Arabia \\ ${ }^{5}$ Department of Pharmacology and Toxicology, Faculty of Pharmacy, Zagazig University, Zagazig, Egypt
}

Correspondence should be addressed to Osama A. A. Ahmed; oaahmed@kau.edu.sa

Received 4 April 2020; Revised 16 June 2020; Accepted 3 July 2020; Published 27 July 2020

Academic Editor: Khalid Rahman

Copyright (C) 2020 Osama A. A. Ahmed et al. This is an open access article distributed under the Creative Commons Attribution License, which permits unrestricted use, distribution, and reproduction in any medium, provided the original work is properly cited.

\begin{abstract}
Multiple risk factors combine to increase the risk of vascular dysfunction in patients suffering from metabolic syndrome (MetS). The current study investigates the extent to which quercetin $(\mathrm{Q})$ and chrysin $(\mathrm{CH})$ protect against vascular dysfunction in MetS rats. MetS was induced by feeding rats a high-salt diet (3\%) and fructose-enriched water (10\%) for 12 weeks. Thoracic aorta was isolated from MetS rats and from control rats, with the latter being injured by methylglyoxal (MG). Aortae were incubated with $\mathrm{CH}$ and $\mathrm{Q}$, and vascular reactivity was evaluated through the analysis of aortic contraction and relaxation in response to $\mathrm{PE}$ and ACh, respectively. The formation of advanced glycation end products (AGEs) and the free radical scavenging activity of 1,1diphenyl-2-picrylhydrazyl (DPPH) were also evaluated following the introduction of $\mathrm{CH}$ and $\mathrm{Q}$. The increased vasoconstriction and impaired vasodilation in MetS aortae were significantly ameliorated by Q and CH. Similarly, they ameliorated glycationassociated exaggerated vasoconstriction and impaired vasodilation produced by $\mathrm{MG}$ in control aortae. In addition, both $\mathrm{Q}$ and $\mathrm{CH}$ were effective in reducing the formation of AGEs and inhibition of glycosylation in response to MG or fructose treatment. Finally, Q successfully scavenged DPPH free radicals while $\mathrm{CH}$ showed significant vasodilation of precontracted aorta that was inhibited by L-NAME. In conclusion, $\mathrm{Q}$ and $\mathrm{CH}$ provide protection against vascular dysfunction in MetS by interfering with AGEs formations and AGEs-associated vascular deterioration, with $\mathrm{CH}$ being largely dependent on NO-mediated mechanisms of vasodilation.
\end{abstract}

\section{Introduction}

The condition "metabolic syndrome (MetS)," describes a group of conditions including central obesity, dyslipidaemia, hyperglycaemia, and hypertension. The presence of all of the mentioned conditions, rather than only a few, is required in order to draw a conclusive diagnosis of MetS [1]. CVD and type 2 diabetes mellitus (T2DM) develop more commonly in individuals who have any of these interrelated metabolic risk factors [2]. The effect of the syndrome upon the population is significant, and as the incidence of MetS is rising, it is important to devise well-defined diagnostic criteria and treatment guidelines.

It is estimated that $37 \%$ of adults in the Kingdom of Saudi Arabia have MetS. The prevalence of the syndrome is lower in rural areas than urban populations, reflecting lifestyle differences in the levels of activity between the two populations [3]. In particular, the rising obesity trend, especially central obesity, is considered a key factor in the development of MetS [4]. Central obesity is associated with 
glucose intolerance, in which the body is less able to use glucose [5]. In MetS, an insulin-resistant state develops, ultimately leading to hyperglycaemia [6].

The adverse side effect of hyperglycaemia upon the cardiovascular system comes from the frequent accompaniment of impaired fibrinolytic pathways and hypercoagulation [7]. Moreover, in MetS, persistent hyperglycaemia promotes glycation, the nonenzymatic reaction between monosaccharide sugars and protein. The glycation of proteins is further heightened by increased quantities of the reactive sugar derivative, methylglyoxal (MG), which is also elevated secondary to hyperglycaemia. This reaction is considered irreversible, producing compounds known as AGEs [8]. AGEs cause blood vessels to become rigid, which, when combined with other pathological manifestations of diabetes, leads to persistent microvascular complications $[9,10]$. Furthermore, as an adjunct to the production of AGEs, the glycoxidation products, dityrosine and $\mathrm{N}^{\prime}$-formyl kynurenine, are formed. These are useful markers that can be quantified to determine the degree of oxidative protein damage $[11,12]$.

Hyperglycaemia is not the only MetS condition that is damaging to the vasculature. Persistent hypertension and hyperlipidaemia also contribute to increased inflammation and oxidative stress and reduced production of the vasodilator, nitric oxide (NO) $[13,14]$. The adverse side effects to vessel function manifest themselves as an attenuation of vasodilation and an increase in vasoconstriction [15]. A number of synthetic pharmaceuticals are used to attenuate vascular dysfunction, but their side effects are undesirable; this highlights the need to identify other effective compounds that are therapeutically safe and do not carry the same adverse side effects. To this end, researchers have investigated a number of naturally occurring compounds, many of which are flavonoids [16].

Two such examples are the flavonol quercetin (Q) and the flavone chrysin $(\mathrm{CH})$. Consistent with other flavonoids, both compounds bear the distinctive tricyclic polyphenolic structure $[17,18]$. Q is a ubiquitous compound, found in many fruits and vegetables including apples, peppers, and onions. On the other hand, sources of $\mathrm{CH}$ are less common but include chamomile, honey, and passionflower (Passiflora caerulea) $[18,19] . \mathrm{Q}$ is reported to offer diverse health benefits, such as anti-inflammation, antioxidation, and the ability to stimulate endothelial production of NO [20-22]. Such reactions are extremely valuable in the prevention and even treatment of grave conditions such as cancer and the wide array of disorders that make up cardiovascular diseases. The established beneficial effects of Q offer a strong basis on which the current research can build. Based on such findings, $\mathrm{Q}$ is a potential compound that could be used to reduce MetS-initiated vascular damage. Similar to $\mathrm{Q}, \mathrm{CH}$ has also been described as offering antioxidant and anti-inflammatory benefits which, as mentioned before, are key reactions involved in the prevention of many diseases. Such benefits of both $\mathrm{Q}$ and $\mathrm{CH}$ have been strongly established, and the results obtained conclude a significantly desirable effect, rather than weak conclusions based on speculation. It must be said however that research indicates that $\mathrm{CH}$ has less pronounce effects when compared to $\mathrm{Q}[23,24]$. Therefore, this study aims to explore the extent to which $\mathrm{CH}$ and $\mathrm{Q}$ offer vascular protection to MetS aorta and the potential mechanisms behind any effects observed.

\section{Materials and Methods}

2.1. Drugs and Chemicals. The following compounds were purchased from Sigma-Aldrich, Dorset, UK: acetylcholine (ACh), aminoguanidine (AG), bovine serum albumin (BSA), chrysin (CH), cinnamaldehyde (CA), diphenyl-2picrylhydrazyl (DPPH), ferulic acid (Fer), fructose (F), methylglyoxal (MG), $\mathrm{N}_{\omega}$-Nitro-L-arginine methyl ester hydrochloride (L-NAME), phenylephrine (PE), and quercetin (Q). CH, Q, and DPPH were dissolved in dimethyl sulfoxide (DMSO) at a concentration of $0.1 \%$ of the reaction media; all other compounds, which were of analytical grade, were dissolved in ultrapure deionized water.

2.2. Experimental Animals. Male Wistar rats aged 7 weeks and weighing between 180 and $200 \mathrm{~g}$ were obtained from King Fahd Medical Research Center (King Abdulaziz University, Jeddah, Saudi Arabia). Groups of four rats were kept in polypropylene animal housing; adequate ventilation, purified water, and standard rodent diet pellets were constantly available. Approval for the study's experimental protocol was granted by the Research Ethical Committee, Faculty of Pharmacy, King Abdulaziz University, Jeddah, Saudi Arabia (approval number 1071439). The Saudi Arabia Research Bioethics and Regulations were observed throughout the study.

To induce MetS, for 12 weeks, the 30 rats were given fructose-enriched water (10\%) and high-salt diet (3\%) [25] while unadulterated food and water were given to control rats. Weight of MetS rats was $250 \mathrm{~g}+$.

2.3. Study Protocol. The rats were randomly allocated to either the control (C) or MetS group. After 12 weeks, the rats were decapitated by rodent guillotine and their descending thoracic aortae harvested. Termination of the rats by guillotine was necessary in order to avoid blood clotting inside the aorta that affects the current experiments.

2.4. Measurements of MetS Indices. Systolic blood pressure (SBP) was measured in control and in rats fed high-fructose and high-salt diet at the end of study by tail cuff method as described in detail in previous work of our laboratories [26]. Briefly, the measurement is preceded by an equilibrating 5-10 min period for the rats in the warming chamber, followed by 10 repetitions of the automated inflation-deflation cycles. Serum insulin level was determined using enzymelinked immunosorbent assay kit using antirat insulin antibodies (Millipore, Billerica, Massachusetts).

2.5. Effect of $Q$ and $\mathrm{CH}$ on MetS Vascular Dysfunction. To determine vascular reactivity, previously established techniques were used to isolate the aorta $[26,27]$. Excised aortae 
from MetS animals were defatted, sectioned into $3 \mathrm{~mm}$ rings, and then suspended in an automated organ bath (Panlab, Barcelona, Spain). In addition to containing a single section of aorta, each channel contained $25 \mathrm{ml}$ of Krebs Henseleit buffer $\left(118 \mathrm{mM} \mathrm{NaCl}, 4.8 \mathrm{mM} \mathrm{KCl}, 2.5 \mathrm{mM} \mathrm{CaCl}_{2}, 1.2 \mathrm{mM}\right.$ $\mathrm{MgSO}_{4}, 1.2 \mathrm{mM} \mathrm{KH}{ }_{2} \mathrm{PO}_{4}, 25 \mathrm{mM} \mathrm{NaHCO}$, and $11.1 \mathrm{mM}$ glucose). The bath was incubated at $37^{\circ} \mathrm{C}$ and continuously aerated with a $95 \% \mathrm{O}_{2}$ and $5 \% \mathrm{CO}_{2}$ gas mixture. Every $30 \mathrm{~min}$, the buffer solution was replaced. To measure aortic tension, an isometric force transducer (ADInstruments, Bella Vista, Australia) was used. Data were collected and recorded using the PowerLab Data Interface Module; this was connected to a PC operating LabChart software v8 (ADInstruments).

To achieve the equilibrium state, aortic tension was adjusted to $1500 \mathrm{mg} \pm 50$ for $20 \mathrm{~min}$. The primary initiator of aortic contraction was PE $\left(10^{-5} \mathrm{M}\right)$, and relaxation was stimulated by ACh $\left(10^{-5} \mathrm{M}\right)$. Once resting-state tension was achieved ( $1500 \mathrm{mg} \pm 50), \mathrm{Q}$ and $\mathrm{CH}$ at concentrations 10 and $30 \mu \mathrm{M}$ were added to the channels and then incubated at $37^{\circ} \mathrm{C}$ for $60 \mathrm{~min}$. The vehicle $(0.1 \% \mathrm{DMSO})$ was added to the control channels. To study the contractile response of the aorta following incubation, PE $\left(10^{-8}\right.$ to $\left.10^{-5} \mathrm{M}\right)$ was $\mathrm{cu}-$ mulatively added. The similar cumulative addition process was applied using $\mathrm{ACh}\left(10^{-8}\right.$ to $\left.10^{-5} \mathrm{M}\right)$ to evaluate the relaxation response but in submaximal $\mathrm{PE}$-precontracted vessels. To establish contraction, tension increase was measured in $\mathrm{mg}$, and, conversely, the percentage of the PEinduced contraction was used to establish relaxation.

\subsection{Effect of Q and CH on MG-Induced Vascular Dysfunction.} For the MG experiment, the same procedure was followed, except that the aorta rings were isolated from control animals and suspended. Then aortic contraction and relaxation were initially stimulated by $\mathrm{PE}$ and $\mathrm{ACh}$, respectively, before being incubated with MG $(100 \mu \mathrm{M})$ in absence or presence of $\mathrm{Q}$ or $\mathrm{CH}(10$ and $30 \mu \mathrm{M})$ at resting tension $(1500 \mathrm{mg} \pm 50)$ for $60 \mathrm{~min}$. Then, as previously described, cumulative concentrations of $\mathrm{PE}$ and $\mathrm{ACh}\left(10^{-8}\right.$ to $\left.10^{-5} \mathrm{M}\right)$ were added.

2.7. Effect of $\mathrm{Q}$ and $\mathrm{CH}$ on AGE Production. 96-well black plate was used to evaluate the effect of $\mathrm{Q}$ and $\mathrm{CH}$ on the formation of AGEs [28]. Bovine serum albumin (BSA), $10 \mathrm{mg} / \mathrm{ml}$ in phosphate buffer saline (PBS), was added first to the wells, followed by the addition of $\mathrm{Q}$ and $\mathrm{CH}$ $(10-100 \mu \mathrm{M})$. AG $(1 \mathrm{mM})$ was used as a glycation inhibitor and was added to the wells containing the BSA and compounds. To this mixture, MG $(50 \mathrm{mM})$ was added directly after preparation and left for incubation in the dark at $37^{\circ} \mathrm{C}$ for $1 \mathrm{~h}$. The same was done with fructose $(50 \mathrm{mM})$ except that the incubation time was 2 weeks and sodium azide $0.02 \%$ was added to all of the wells. A row of wells was kept as control where PBS was added instead of MG or fructose. To establish the production AGEs, the fluorescence intensity was measured at $\lambda \mathrm{ex}=325$ and $\lambda \mathrm{em}=440 \mathrm{~nm}$ using a Monochromator SpectraMax ${ }^{\circledR}$ M3 plate reader (Molecular Devices, Sunnyvale, CA, USA). To measure the quantities of dityrosine, $\mathrm{N}^{\prime}$-formyl kynurenine, and kynurenine, fluorescence intensity was measured at $\lambda \mathrm{ex}=330,325$, and $365 \mathrm{~nm}$, and $\lambda \mathrm{em}=415,434$, and $480 \mathrm{~nm}$ [29].

2.8. Antioxidant Activity of $\mathrm{Q}$ and $\mathrm{CH}$. The compound's antioxidant potential or ability to scavenge reactive oxygen species (ROS) was researched (with modification) as described by [30]. In a 96-well clear plate, $\mathrm{Q}$ and $\mathrm{CH}$ $(10-100 \mu \mathrm{M})$ in methanol were added with the blank kept as only methanol. DPPH solution $(240 \mu \mathrm{M})$ in methanol/tris $(1: 1 \mathrm{v} / \mathrm{v})$, prepared immediately prior to use, was then added to the wells. The same concentrations of $\mathrm{Q}$ and $\mathrm{CH}$ were added to only methanol/tris $(1: 1 \mathrm{v} / \mathrm{v})$ for control. Using the Monochromator SpectraMax ${ }^{\circledR}$ M3 plate reader (Molecular Devices, Sunnyvale, CA, USA), the absorbance was measured every minute for $10 \mathrm{~min}$ at $520 \mathrm{~nm}$.

2.9. Direct Relaxation Effect of $Q$ and $\mathrm{CH}$. In accordance with the procedure described earlier, aorta rings were suspended in KHB-filled channels. They were kept at resting tension using the same method previously described, tested for $\mathrm{PE}$ contraction and $\mathrm{ACh}$ relaxation, and then directly incubated at $37^{\circ} \mathrm{C}$ for $30 \mathrm{~min}$ with the nitric oxide synthase inhibitor, L-NAME (1 mM). A submaximal dose of PE was added to initiate preconstriction; once contraction plateaued, ascending concentrations $(10-100 \mu \mathrm{M})$ of $\mathrm{CH}$ and $\mathrm{Q}$ were added. Before the addition of the subsequent concentration, the tissue was given time to reach the relaxation plateau. The vehicle was added to time control channels to eliminate any possible effect of DMSO whose final concentration did not exceed $0.1 \%$. Before termination of the experiment, a single dose of ACh $10^{-5} \mathrm{M}$ was added to all of the channels to achieve the complete aortic relaxation.

2.10. Statistical Analysis. One-way analysis of variance (ANOVA) followed by Dunnett's post hoc test was used to analyze the glycation experiment data. Two-way ANOVA followed by Bonferroni post hoc test was used for the vascular reactivity, free radical scavenging activity, and direct relaxation experiments. Unpaired Student's $t$-test was conducted to analyze MetS data. These analyses were carried out using GraphPad Instant software, version 5 (GraphPad Software, Inc., La Jolla, CA, USA). Statistical significance was set at $p<0.05$. Experimental values were expressed as mean \pm SEM (standard error of the mean).

\section{Results}

3.1. Effect of High-Fructose High-Salt Diet on MetS Indices. Feeding rats on high-fructose (10\% in drinking water) and high-salt diet (3\%) for 12 weeks led to development of metabolic syndrome in these animals as indicated by the significant elevations in systolic blood pressure and serum insulin and increase in body weight (Table 1).

\subsection{Effect of $Q$ and $\mathrm{CH}$ on MetS Vascular Dysfunction.} Compared to control, there was considerably greater vascular contraction in the MetS aorta in response to $\mathrm{PE}\left(10^{-8}\right.$ to 
TABLE 1: Effect of high-fructose (10\%) high-salt (3\%) feeding for 12 weeks on the increase in systolic blood pressure (SBP), body weight, and serum insulin in rats.

\begin{tabular}{lccc}
\hline Treatment & SBP (mmHg) & Body weight $(\mathrm{g})$ & $\begin{array}{c}\text { Serum insulin } \\
(\mathrm{ng} / \mathrm{dl})\end{array}$ \\
\hline Control & $115.2 \pm 3.9$ & $285.8 \pm 10.4$ & $2.32 \pm 0.33$ \\
MetS & $134.5^{*} \pm 6.7$ & $356.2^{*} \pm 16.8$ & $6.93^{*} \pm 0.97$ \\
\hline
\end{tabular}

Values are expressed as mean $\pm \mathrm{SEM}$; ${ }^{*} p<0.05$, compared with the corresponding control group values using unpaired $t$-test. $N=6$ rats.

$10^{-5} \mathrm{M}$ ) (Figure 1 ). This is indicated by the higher tension in the MetS aorta starting from around $10^{-5.5} \mathrm{M}$ PE concentration and reaching a maximum effect at $142 \%$ of the control values. Incubation of the MetS aorta with 10 and $30 \mu \mathrm{M}$ of $\mathrm{Q}$ or $\mathrm{CH}$ significantly reduced contraction in a concentration-dependent manner.

As the data in Figures 2(a) and 2(b) demonstrate, vascular relaxation in the MetS aorta was impaired in response to ACh $\left(10^{-8}\right.$ to $\left.10^{-5} \mathrm{M}\right)$ compared to the control. There was a significant difference between the MetS aorta and the control, with the effect appearing at approximately $10^{-6.5} \mathrm{M}$ $\mathrm{ACh}$ and reaching a maximum relaxation at $72 \%$ of the control values. Incubating the MetS aorta with 10 and $30 \mu \mathrm{M}$ of $\mathrm{Q}$ or $\mathrm{CH}$ corrected the attenuated vasorelaxation to control levels. The graphs show that these concentrations of the compounds were similarly effective in lowering the aorta tension during relaxation.

\subsection{Effect of $Q$ and $C H$ on MG-Induced Vascular} Dysfunction. As Figures 3(a) and 3(b) show, the addition of MG to the aorta caused exaggerated vascular contraction in response to $\mathrm{PE}\left(10^{-8}\right.$ to $\left.10^{-5} \mathrm{M}\right)$ when compared to control. This is demonstrated by the tension in MG-treated aorta being significantly higher, starting from $10^{-6.5} \mathrm{M}$ PE concentration. Both concentrations of Q and $\mathrm{CH}(10$ and $30 \mu \mathrm{M})$ significantly reduced the extent of contraction, returning it back to normal and, in the case of $\mathrm{CH}$, even below that of the control.

Figures 3(c) and 3(d) show that, following incubation with MG, smooth muscle dilatation of the aorta in response to ACh $\left(10^{-8}\right.$ to $\left.10^{-5} \mathrm{M}\right)$ was impaired compared to control. As portrayed in Figures 3(c) and 3(d), there was a significant difference between the MG-treated aorta and the control during vascular relaxation, with the effect appearing at approximately $10^{-7} \mathrm{M}$ ACh. Both concentrations of $\mathrm{Q}$ and $\mathrm{CH}(10$ and $30 \mu \mathrm{M})$ were able to ameliorate this impaired vasorelaxation, restoring it to values that were comparable to those of control.

3.4. Effect of Q and CH on AGE Production. Figures 4(a)-4(c) show that, compared with the control, incubation of BSA $(10 \mathrm{mg} / \mathrm{ml})$ with $\mathrm{MG}(50 \mathrm{mM})$ resulted in a significant increase in AGE production, as well as the protein oxidation products, dityrosine, kynurenine, and $\mathrm{N}$-formyl kynurenine. Addition of $A G(1 \mathrm{mM})$ in the reaction mixture significantly suppressed the levels of AGEs and the protein oxidation products. Both concentrations of $\mathrm{Q}$ and $\mathrm{CH}$ were significantly able to inhibit the formation of MG-mediated AGEs and resultant protein oxidation in a concentrationdependent manner.

A similar pattern of results is seen for the fructosemediated glycation reaction (Figures 5(a)-5(c)). Incubating BSA with $\mathrm{F}(50 \mathrm{mM})$ resulted in a significant increase in AGE and protein oxidation products compared with the control. This was significantly inhibited by AG (1 mM). With the exception of $10 \mu \mathrm{M}$ of $\mathrm{Q}$, levels of $\mathrm{AGE}$ and protein oxidation were significantly reduced by all concentrations of $\mathrm{CH}$ and $\mathrm{Q}$.

3.5. Free-Radical Scavenging Activity of $Q$ and $\mathrm{CH}$. Figures 6(a) and 6(b) display the results of the $10 \mathrm{~min}$ reaction between DPPH and the natural compounds. The results indicate that only $\mathrm{Q}$ possessed $\mathrm{DPPH}$ free radical scavenging activity, which is translated into an antioxidant effect. Figure 6(a) shows that both concentrations of $Q$ operate in a concentration-dependent manner significantly influencing antioxidant activity. Furthermore, the graph reveals that that the reaction took place within the first few minutes and then reached a plateau. The time taken to reach that plateau varied according to the concentration used. As expected, a faster reaction was seen with the $30 \mu \mathrm{M}$ Q, lasting for 6 minutes, compared to a 10 minute reaction time when $\mathrm{Q} 10 \mu \mathrm{M}$ was added.

3.6. Direct Relaxation Effect of $Q$ and $C H$. Following a single dose of $\mathrm{PE}\left(10^{-5} \mathrm{M}\right)$ to induce contraction, the addition of cumulative concentrations of $\mathrm{Q}$ (at 10 and $30 \mu \mathrm{M}$ ) to the aorta did not have any effect (Figure 7(a)). However, addition of cumulative concentrations of $\mathrm{CH}$ (at 10 and $30 \mu \mathrm{M})$ to the aorta brought about a decrease in tension and hence concentration-dependent vasodilation $(p<0.05$, Figure $7(\mathrm{~b}))$. $\mathrm{CH}$ produced potent vasodilation that reached $90 \%$ relaxation at concentration of $30 \mu \mathrm{M}$ of $\mathrm{CH}$. In addition, the aorta incubated with L-NAME (1 mM) completely blocked the mentioned $\mathrm{CH}$ potent vasodilation $(p<0.05$, Figure $7(\mathrm{~b}))$.

\section{Discussion}

The purpose of this study was to investigate whether $Q$ and $\mathrm{CH}$ possess vasculoprotective effects to ameliorate vascular damage commonly seen in MetS and to determine possible mechanisms of action. To our knowledge, this is the first study to investigate the direct vasculoprotective effects of the natural compounds $\mathrm{Q}$ and $\mathrm{CH}$ on MetS aorta and the role of AGE inhibition in their effects. The results showed that $Q$ and $\mathrm{CH}$ protect against MetS associated vascular dysfunction. The common effect of $\mathrm{Q}$ and $\mathrm{CH}$ compounds was to (1) interfere with AGEs-induced exaggerated vasoconstriction and impaired vasodilation and (2) significantly inhibit AGEs forming in a dose-dependent manner. In addition, $\mathrm{Q}$ had extra free radical scavenging activity, while $\mathrm{CH}$ showed nitric oxide-dependent vasodilation.

Vascular dysfunction is associated with several MetSrelated factors, the most important of which are the 


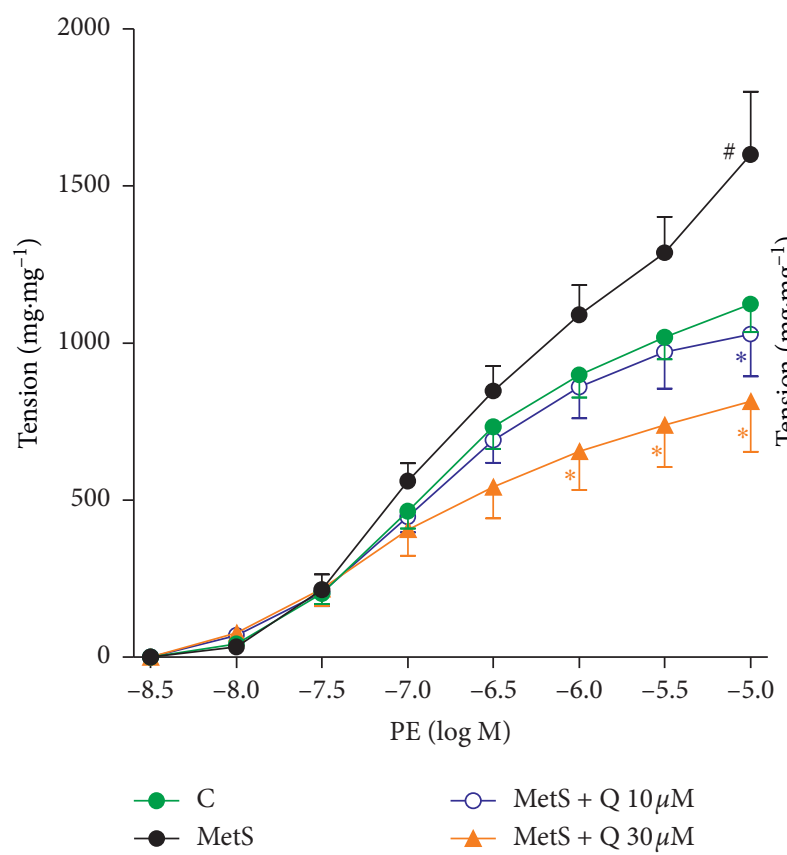

(a)
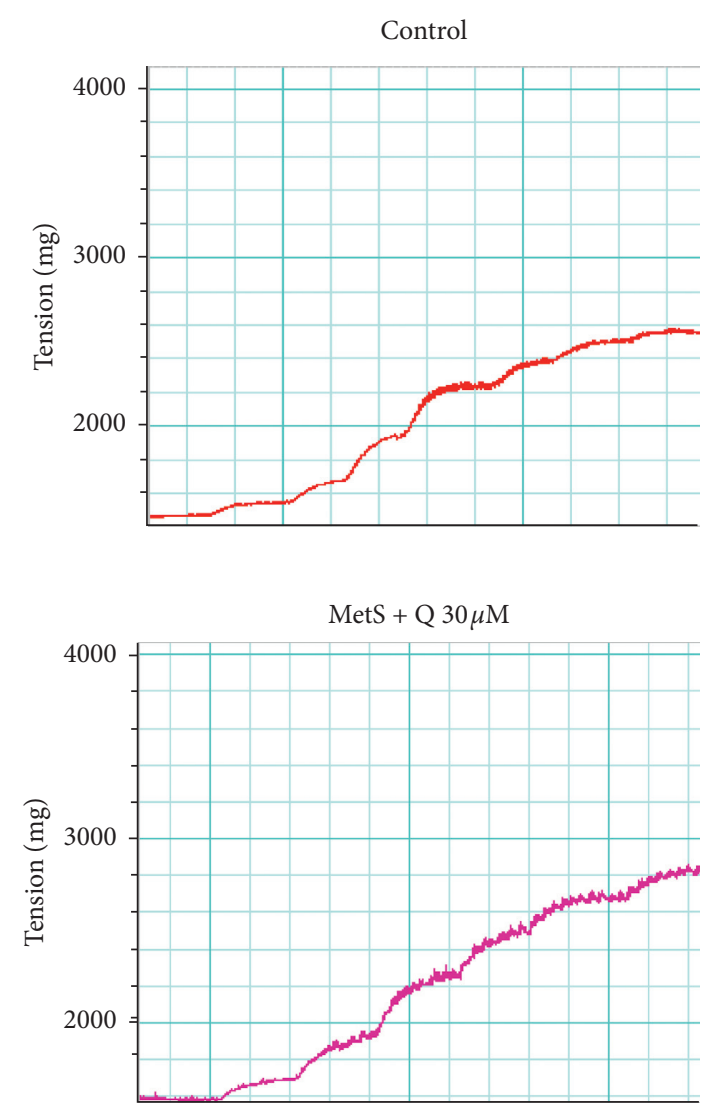

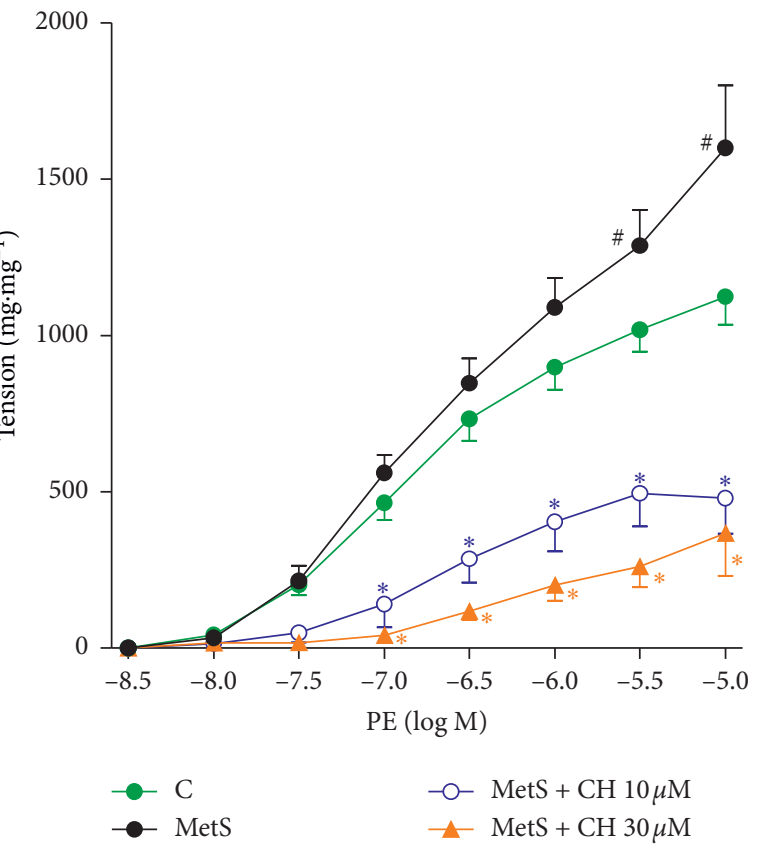

(b)
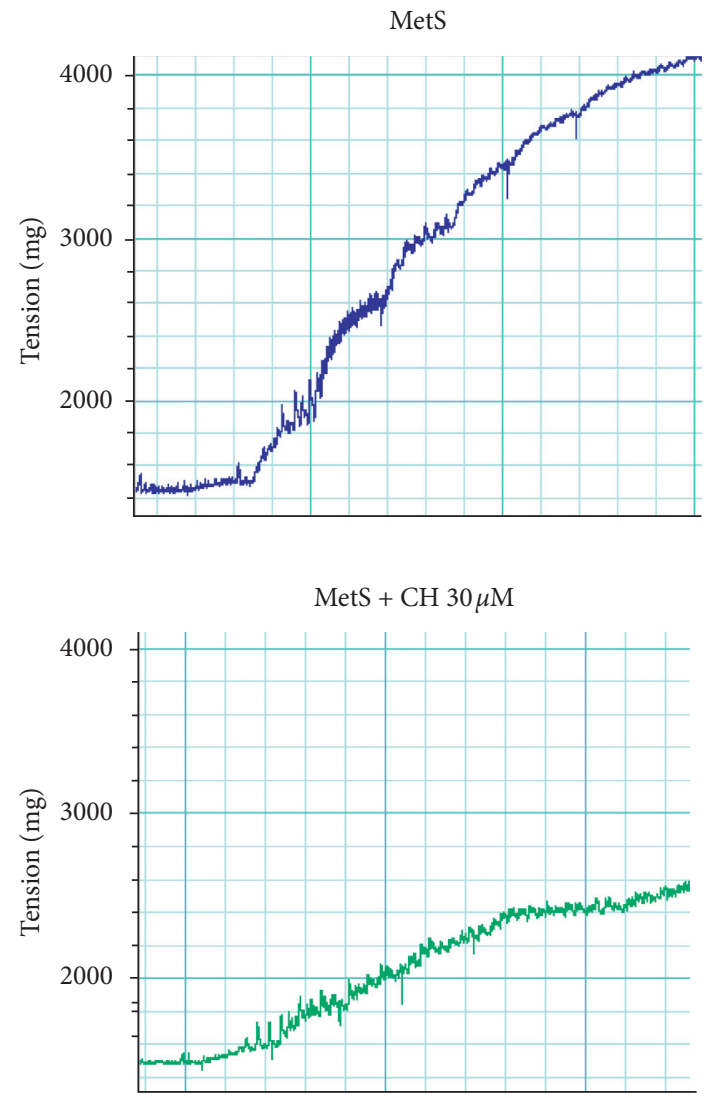

(c)

Figure 1: Effect of (a) Q and (b) $\mathrm{CH}$ on isolated aorta responsiveness to $\mathrm{PE}$ in fructose-induced MetS after incubation with Q or $\mathrm{CH}$ at $37^{\circ} \mathrm{C}$ for 60 minutes. Results are expressed as mean \pm SEM $(n=6-8) .{ }^{\#} p<0.05$ when compared to control, ${ }^{*} p<0.05$ when compared to MetS by two-way ANOVA followed by Bonferroni post hoc test. (c) Representative recording charts of control, MetS, MetS $+\mathrm{Q} 30 \mu \mathrm{M}$, and MetS $+\mathrm{CH} 30 \mu \mathrm{M}$ experiments. 


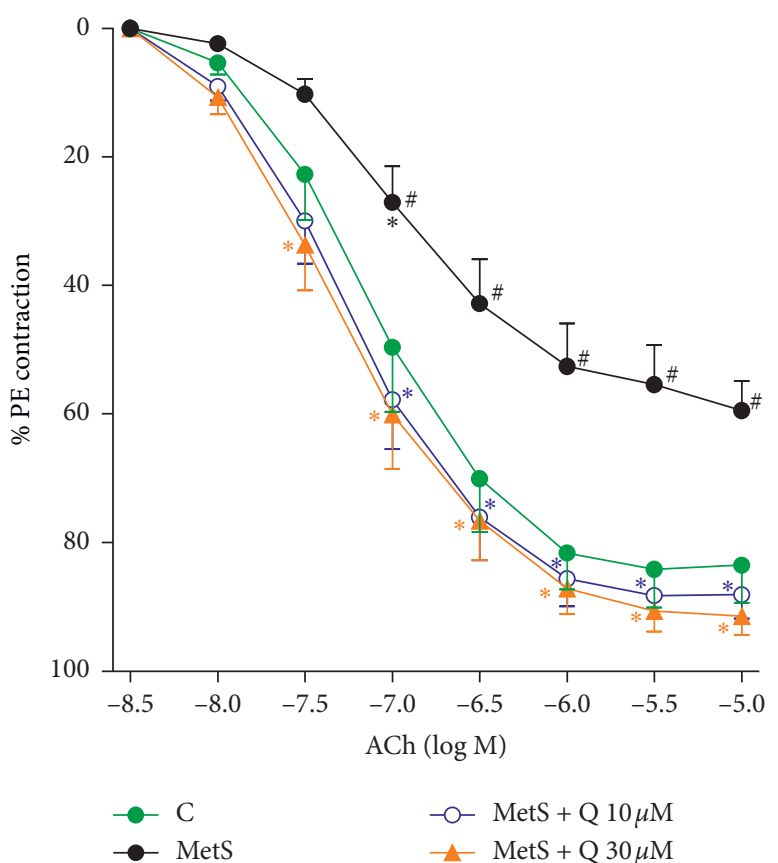

(a)

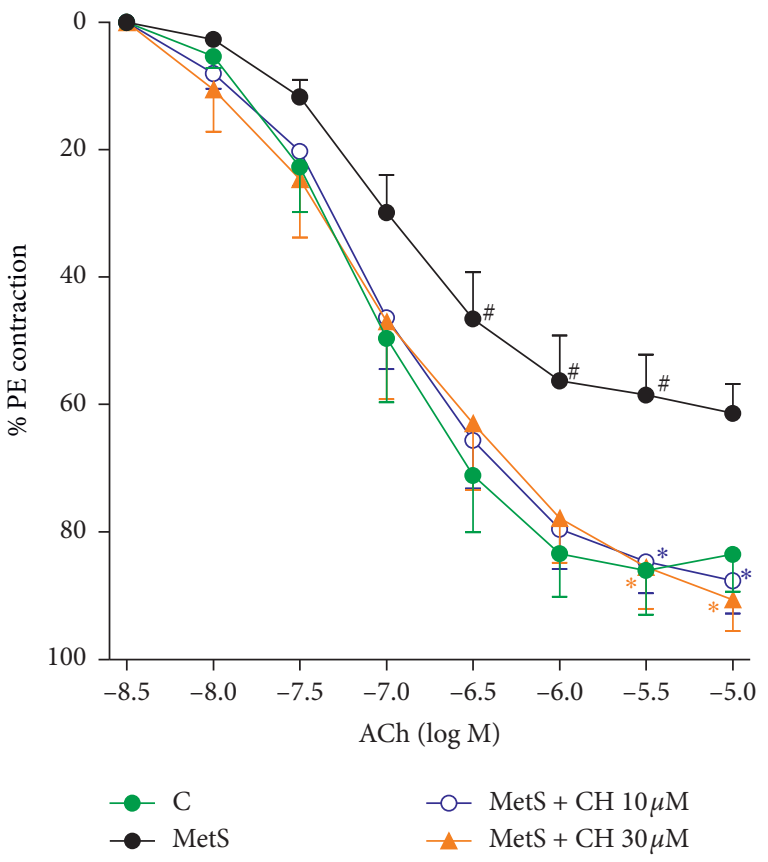

(b)

Figure 2: Effect of (a) Q and (b) $\mathrm{CH}$ on isolated aorta responsiveness to $\mathrm{ACh}$ in fructose-induced MetS after incubation with Q or $\mathrm{CH}$ at $37^{\circ} \mathrm{C}$ for 60 minutes. Results are expressed as mean $\pm \operatorname{SEM}(n=6-8) .{ }^{\#} p<0.05$ when compared to control, ${ }^{*} p<0.05$ when compared to MetS by two-way ANOVA followed by Bonferroni post hoc test.

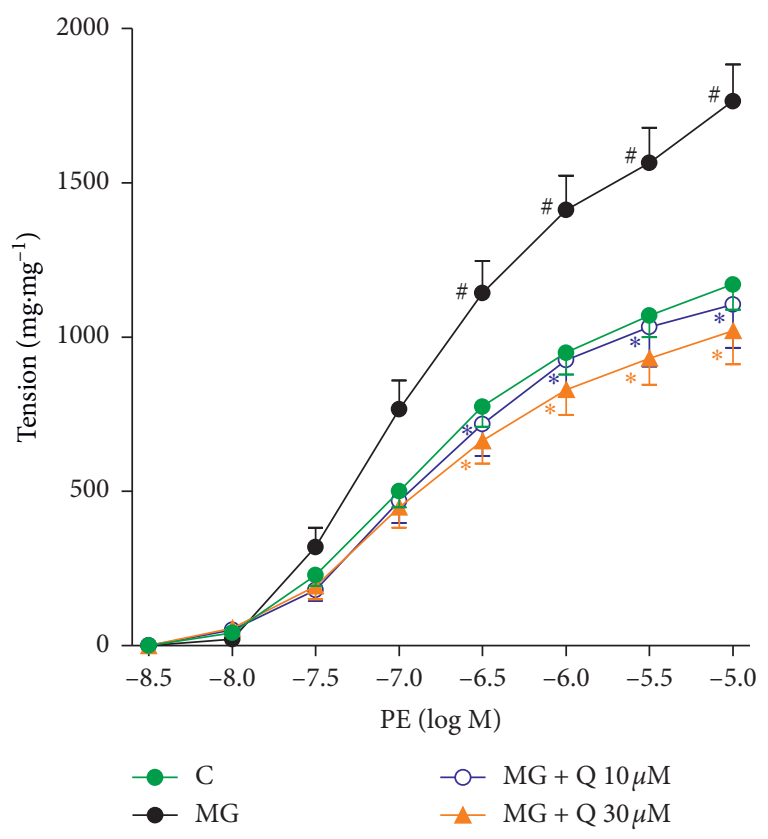

(a)

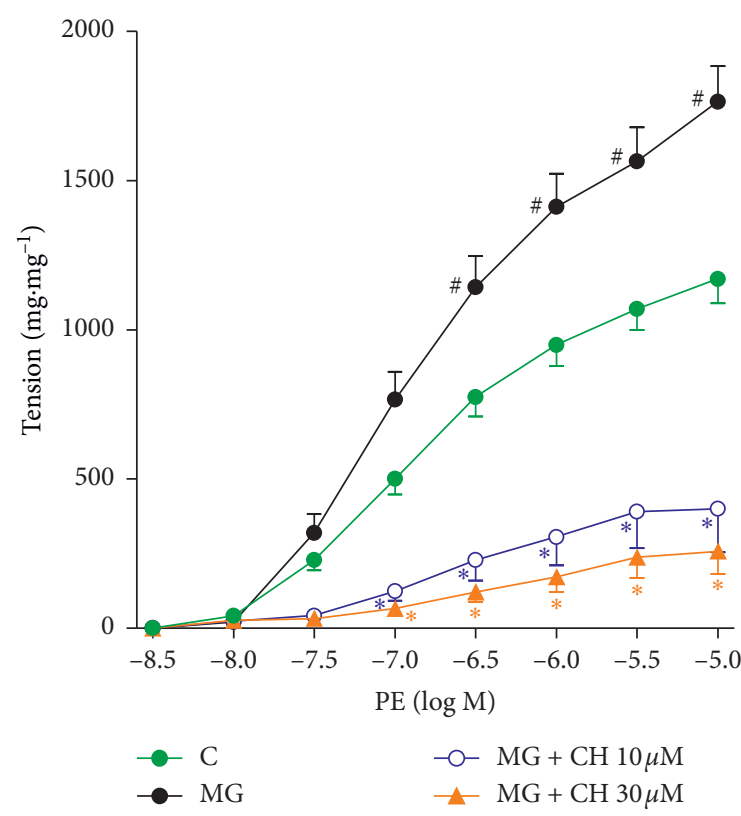

(b)

FIGURE 3: Continued. 


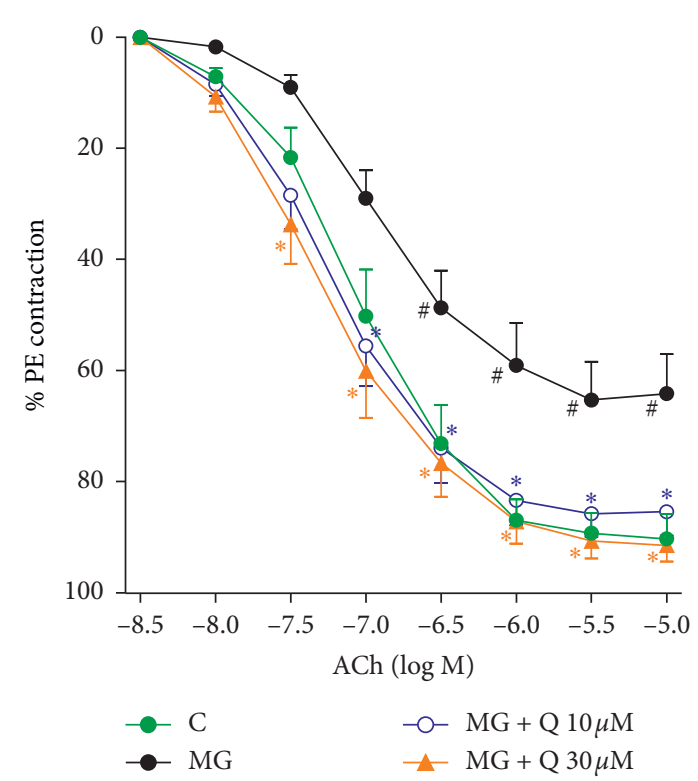

(c)

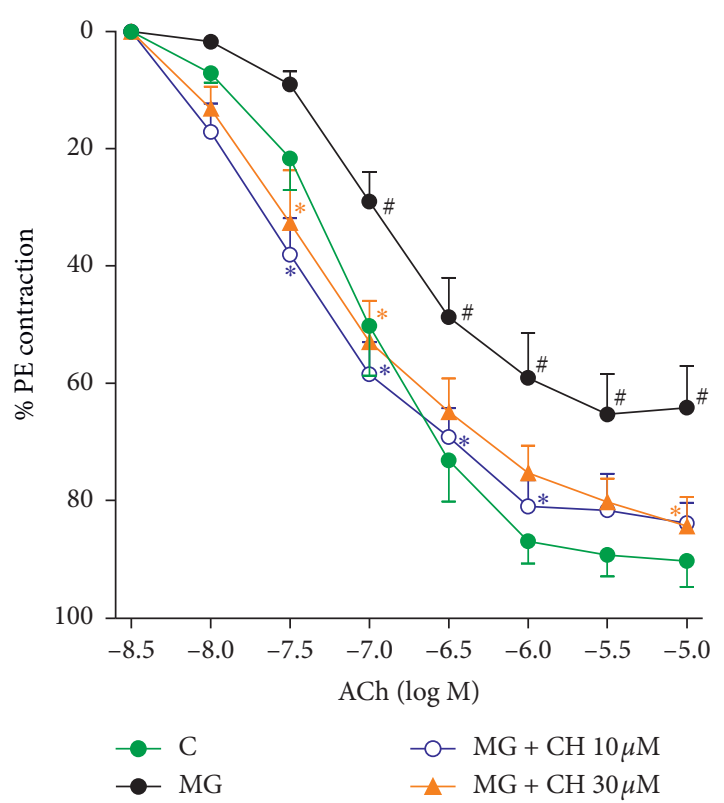

(d)

FIGURE 3: Effect of (a) Q and (b) $\mathrm{CH}$ on isolated normal aorta responsiveness to PE after incubation with $\mathrm{MG}$ (100 $\mu \mathrm{M})$ in the presence and absence of $\mathrm{Q}$ or $\mathrm{CH}$ at $37^{\circ} \mathrm{C}$ for 60 minutes. Effect of (c) Q and (d) $\mathrm{CH}$ on isolated normal aorta responsiveness to ACh after incubation with $\mathrm{MG}(100 \mu \mathrm{M})$ in the presence and absence of $\mathrm{Q}$ or $\mathrm{CH}$ at $37^{\circ} \mathrm{C}$ for 60 minutes. Results are expressed as mean $\pm \mathrm{SEM}(n=6-8){ }^{\#} p<0.05$ when compared to control, ${ }^{*} p<0.05$ when compared to MG by two-way ANOVA followed by Bonferroni post hoc test.

advanced glycation end products (AGEs) [31]. Such vascular dysfunction is characterized by increased vasoconstriction and attenuated dilatation [15]. Other than MetS, vascular dysfunction can be directly induced in vitro with the use of the glycation intermediate methylglyoxal (MG). Being a highly reactive sugar derivative, $M G$ can result in acute production of AGEs, which is largely responsible for vascular damage. This finding is in agreement with that of Dhar et al. [32] who proved that MG results in vascular dysfunction characterized by attenuated ACh-induced aortic relaxation.

The results show that $\mathrm{Q}$ and $\mathrm{CH}$ significantly alleviated the exaggerated vasoconstriction of the aortic rings and attenuated ACh-induced vasodilation found in MetS aortae. This finding is consistent with that of Sánchez et al. [33] who demonstrated that in vivo treatment with $\mathrm{Q}$ in hypertensive rats enhanced aortic vasodilation. In addition, El-Bassossy et al. [34] also proved the ability of $\mathrm{CH}$ treatment to improve the exaggerated vasoconstriction in insulin-resistant rats.

The effect on AGEs-induced vascular damage was investigated as a possible mechanism of action of $\mathrm{Q}$ and $\mathrm{CH}$. In this regard, both $\mathrm{Q}$ and $\mathrm{CH}$ inhibited MG-induced exaggerated vasoconstriction and impaired vasodilation in a very similar way to that observed in MetS aortae. This suggests that AGEs can be a common important pathway for Q and $\mathrm{CH}$ vascular protection effect.

The effect on AGEs formation was studied in order to further investigate the effect of $\mathrm{Q}$ and $\mathrm{CH}$ on different sides of the AGEs pathways. The current study showed that both $\mathrm{Q}$ and $\mathrm{CH}$ significantly inhibit both fructose- and MGproduced AGEs in a concentration-dependent manner. One hypothesized mechanism for Q's antiglycation action is that the compound has a polyphenolic structure, enabling it to scavenge free radicals. The structure, which is abundant with hydroxyl groups, is essential to the antioxidant effect shown by the compounds [35]. Such compounds donate a hydrogen atom to reduce free radicals, hence preventing protein oxidation, a main step in AGE formation. This is further proven by the strong antioxidant activity of $Q$ in its reaction with the DPPH free radical. This is consistent with several previously conducted studies, which have researched the antiglycation activity of Q [36-38].

Regarding the flavonoid $\mathrm{CH}$, its inability to scavenge DPPH free radicals has been investigated by Kang et al. [39], who reported that $\mathrm{CH}$ is missing two hydroxyl groups, which are essential for the compound (flavone) to exhibit antioxidant activity. This is further proven by Naso et al. [40], who stated that $\mathrm{CH}$ was unable to diminish the levels of $\mathrm{DPPH}$ radicals. However, despite $\mathrm{CH}$ possessing lower antioxidant potential than flavonoids such as Q, it is still able to prevent protein glycation significantly. This finding coincides with that of Matsuda et al. [41] who stated that various flavonoids with strong AGE formation inhibition may be poor scavengers of DPPH radicals. A suggested mechanism of action of $\mathrm{CH}$ in inhibiting the formation of AGEs could be the strong affinity of the flavone to BSA, which prevents the protein's glycation [42]. The ability of $\mathrm{CH}$ to decrease AGE levels has also been demonstrated in vivo where the flavonoid significantly inhibited the increase in serum AGEs in diabetic animals [34].

A mechanism of action for such an effect might be that, in response to $\mathrm{CH}$ and $\mathrm{Q}, \mathrm{NO}$ is released from endothelial tissue, directly causing PE-precontracted aorta to relax significantly. Evidence in support of this NO-dependent 

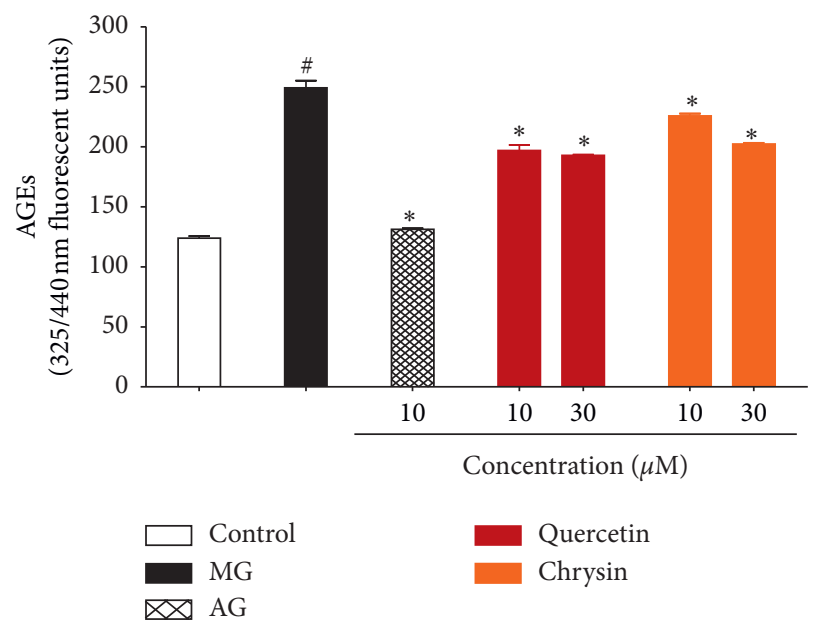
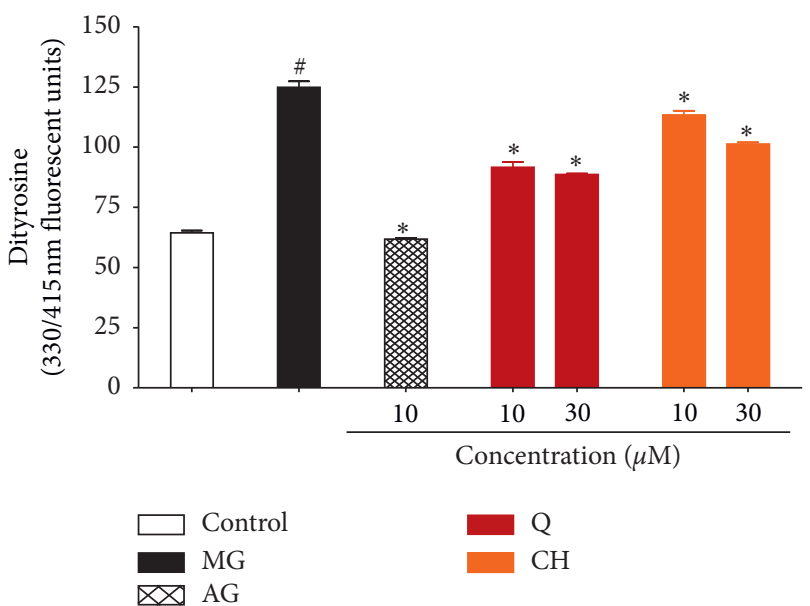

(a)

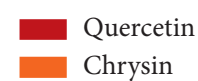

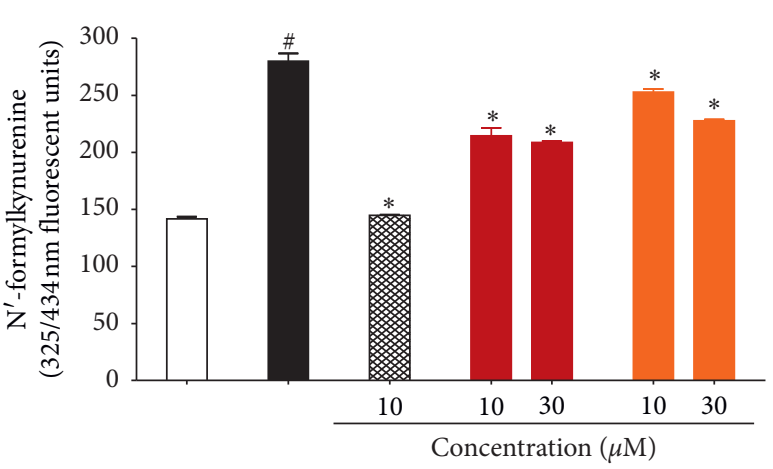

(b)
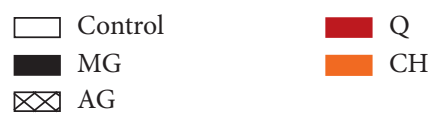

(c)

Figure 4: Effect of Q and CH on (a) AGE, (b) dityrosine, and (c) N-formyl kynurenine production upon incubation of BSA (10 mg/ml) with MG $(50 \mathrm{mM})$ at $37^{\circ} \mathrm{C}$ for one hour. Control is a reaction mixture including only BSA while the MG reaction mixture consists of BSA with MG. AG was used as positive control. Results are expressed as mean $\pm \operatorname{SEM}(n=3)$. ${ }^{\#} p<0.05$ when compared to control, ${ }^{\#} p<0.05$ when compared to MG by one-way ANOVA followed by Dunnett's post hoc test.
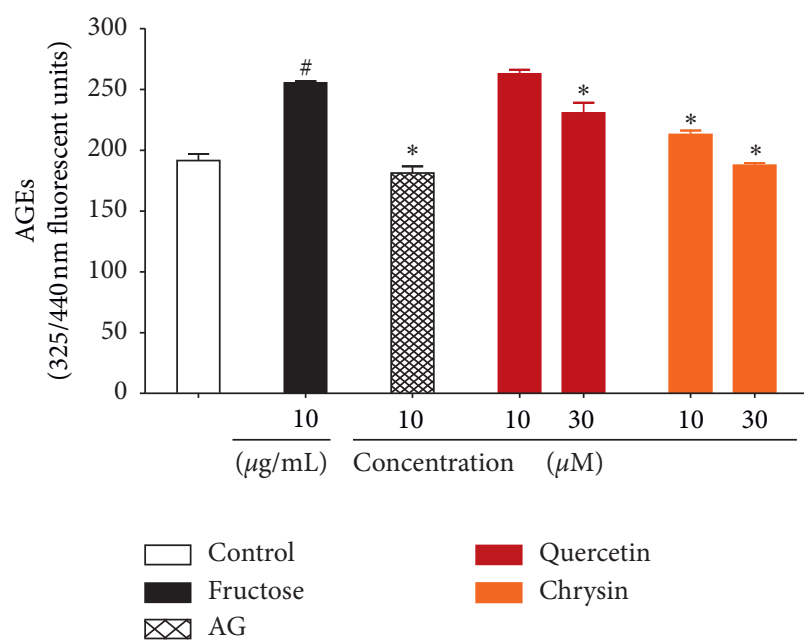

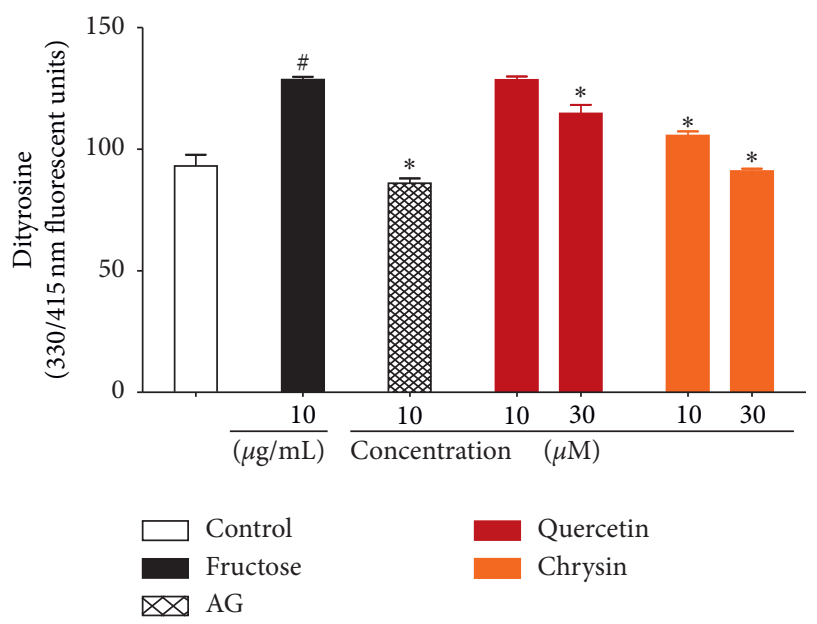

(b)

Figure 5: Continued. 


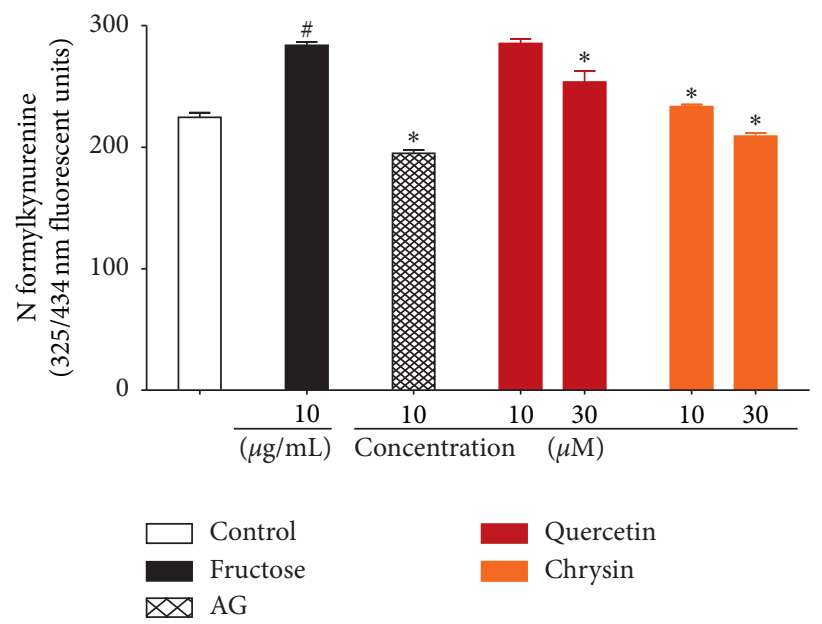

(c)

Figure 5: Effect of $\mathrm{Q}$ and $\mathrm{CH}$ on (a) AGE, (b) dityrosine, and (c) $\mathrm{N}$-formyl kynurenine production upon incubation of BSA (10 mg/ml) with $\mathrm{F}(50 \mathrm{mM})$ at $37^{\circ} \mathrm{C}$ for two weeks. Control is a reaction mixture including only BSA while the F reaction mixture consists of BSA with F. AG was used as positive control. Results are expressed as mean $\pm \operatorname{SEM}(n=3) .{ }^{\#} p<0.05$ when compared to control, ${ }^{*} p<0.05$ when compared to F by one-way ANOVA followed by Dunnett's post hoc test.

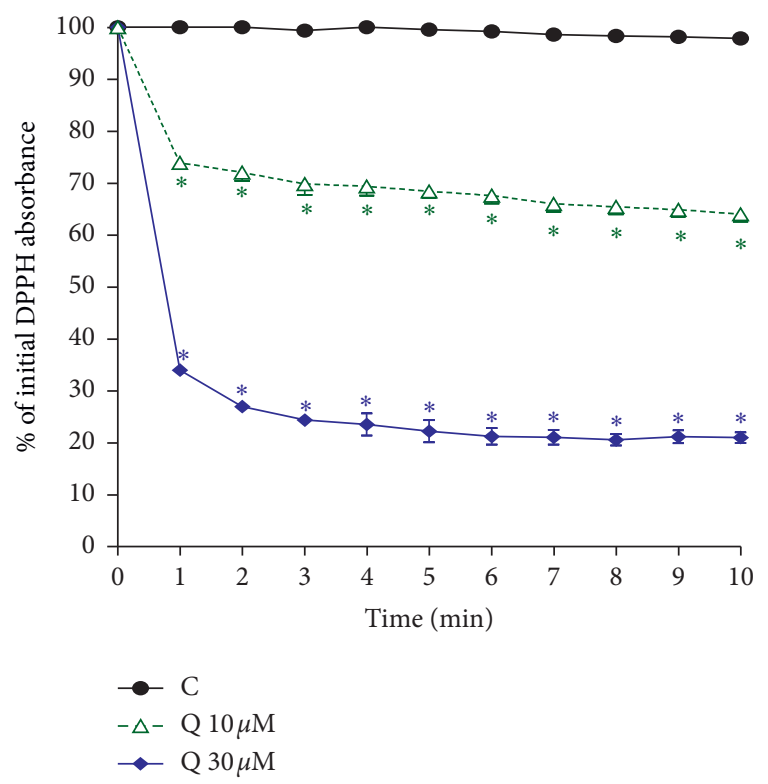

(a)

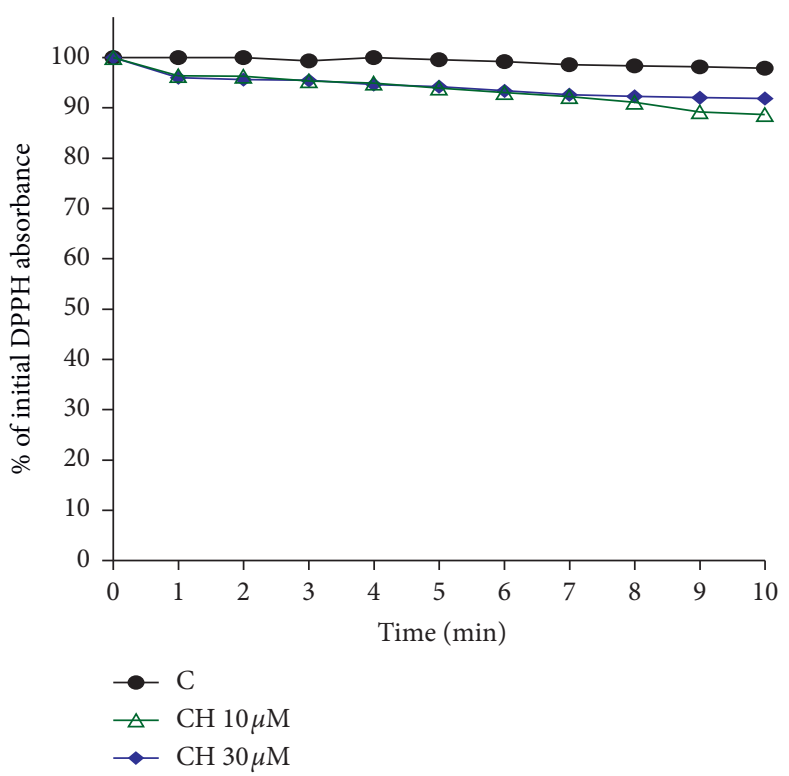

(b)

FIGURE 6: Effect of (a) Q and (b) CH on ROS production as initiated by DPPH ( $240 \mu \mathrm{M})$. Control is a reaction mixture including only DPPH. Results are expressed as mean \pm SEM $(n=3)$. ${ }^{*} p<0.05$ when compared to each corresponding control by two-way ANOVA followed by Bonferroni post hoc test.

mechanism comes from the compounds' direct relaxation effect on precontracted aorta, which was significantly reduced in the presence of the eNOS inhibitor L-NAME. A closer observation of the results shows that both the MGtreated aorta and the $\mathrm{CH}$-treated MetS aorta display vasoconstriction much lower than that seen in control animals. This effect was unique for $\mathrm{CH}$ rather than Q. Furthermore, L-NAME inhibited a direct relaxation effect of $\mathrm{CH}$ more than that of Q. Both these findings may suggest that $\mathrm{CH}$ in particular causes potent eNOS stimulation producing greater levels of NO when compared to Q. This is in accordance with the findings of Villar et al. [43] who concluded that $\mathrm{CH}$, unlike several other flavonoids, brought about aortic relaxation in vitro mainly through endothelium-dependent methods involving increased endothelial NO production. Duarte et al. [44] further argued that $\mathrm{CH}$ may in fact induce vascular relaxation in isolated aorta through additional NO-related mechanisms other than 


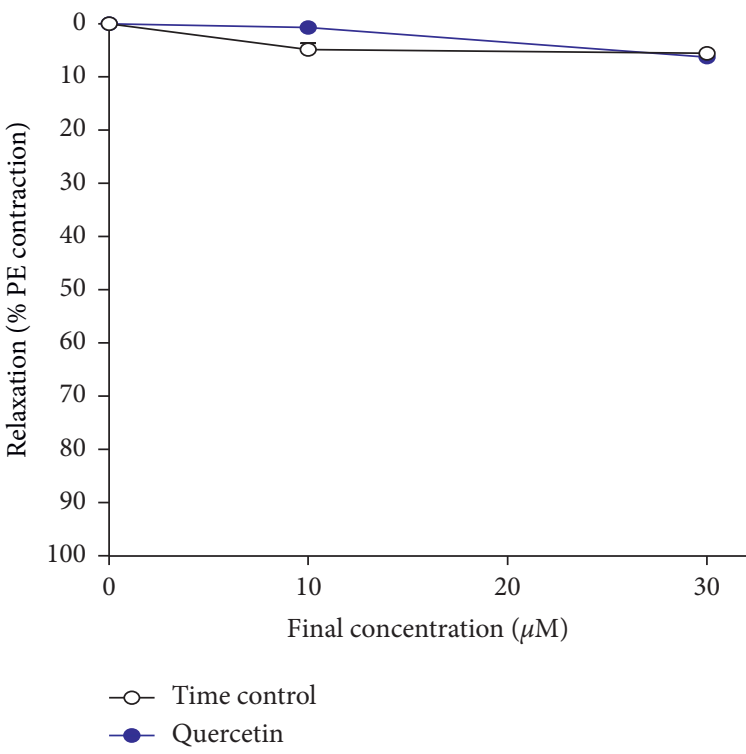

(a)

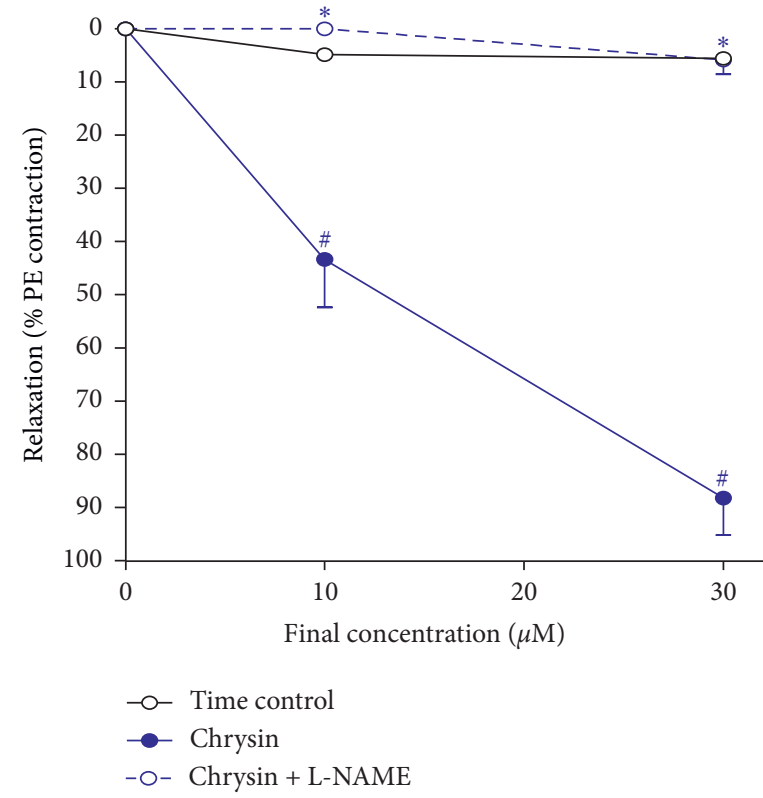

(b)

Figure 7: Direct vasorelaxation effect of the compounds (a) Q and (b) $\mathrm{CH}$ on PE-precontracted isolated aorta with and without incubation with L- NAME $(1 \mathrm{mM})$ at $37^{\circ} \mathrm{C}$ for 30 minutes. Results are expressed as mean \pm SEM $(n=6-8) .{ }^{*} p<0.05$ when compared to control by twoway ANOVA followed by Bonferroni post hoc test.

eNOS stimulation. The study proved that $\mathrm{CH}$ was able to improve the response of the aorta to authentic NO through scavenging superoxide anions (O2•-) which broke down NO. In addition, $\mathrm{CH}$ also potentiated the cGMP pathway, signaling cascade involved in NO-induced vasodilation. This was proven by previous studies which reported an increase in cGMP accumulation in isolated rat aorta by $\mathrm{CH}$ (Villar et al. 2005).

The results of this study prove that, apart from the NOreleasing mechanism, both $\mathrm{Q}$ and $\mathrm{CH}$ protect aorta sections from vascular damage by preventing AGEs from forming. The AGEs alter the structure of proteins and their functions. In the case of blood vessels, glycated collagen results in reduced vessel elasticity [45]. In addition, elevated levels of AGE increase oxidative stress, activate inflammatory mediators, alter the lipid profile, and quench NO, all of which contribute to endothelial damage [46].

The obvious effect of these compounds on ameliorating vascular dysfunction, as proven by this study, can be used as a basis for future research. In addition, the possible effects that the compounds may have in vivo, when added to the diet, and the possible improvement in the same parameters measured can be further investigated.

\section{Conclusions}

The current study shows that the natural flavonoid compounds $\mathrm{Q}$ and $\mathrm{CH}$ have direct vasculoprotective effects on isolated thoracic aorta from MetS rats. This is demonstrated by the compounds' ability to ameliorate the exaggerated vasoconstriction and attenuated vasodilation typical of MetS vascular dysfunction. This effect can be attributed to the compounds' ability to reduce AGE production as well as the concomitant protein oxidation products. In addition, there is significant dependence on NO-mediated mechanisms and, in the case of $\mathrm{Q}$, antioxidant activity, which contribute to the vascular protection.

$\begin{array}{ll}\text { Abbreviations } \\ \text { ACh: } & \text { Acetylcholine } \\ \text { AGEs: } & \text { Advanced glycation end products } \\ \text { AG: } & \text { Aminoguanidine } \\ \text { ANOVA: } & \text { Analysis of variance } \\ \text { BSA: } & \text { Bovine serum albumin } \\ \text { Q: } & \text { Quercetin } \\ \text { CH: } & \text { Chrysin } \\ \text { CVD: } & \text { Cardiovascular diseases } \\ \text { DPPH: } & \text { Diphenyl-2-picrylhydrazyl } \\ \text { L-NAME: } & \text { N } \omega \text {-Nitro-L-arginine methyl ester } \\ & \text { hydrochloride } \\ \text { MetS: } & \text { Metabolic syndrome } \\ \text { PE: } & \text { Phenylephrine } \\ \text { MG: } & \text { Methylglyoxal } \\ \text { NO: } & \text { Nitric oxide } \\ \text { PBS: } & \text { Phosphate buffer saline } \\ \text { T2DM: } & \text { Type 2 diabetes mellitus } \\ \text { SBP: } & \text { Systolic blood pressure. }\end{array}$

\section{Data Availability}

The data used to support the findings of this study are available from the corresponding author upon request. 


\section{Conflicts of Interest}

The authors declare that they have no conflicts of interest regarding the publication of this paper.

\section{Authors' Contributions}

OAAA raised the idea and revised the manuscript; ASA shared the idea and revised the manuscript; MMTperformed the experiments and drafted the manuscript; KSB shared the idea and revised the manuscript; and HEB shared the idea, analyzed the results, and revised the manuscript.

\section{Acknowledgments}

The authors would like to thank Mr. Islam Farouk, Department of Pharmacology and Toxicology, Faculty of Pharmacy, King Abdulaziz University, Jeddah, Saudi Arabia, for his technical support. This work was supported by the National Science, Technology and Innovation Plan (NSTIP) Strategic Technologies Program in the Kingdom of Saudi Arabia, Project No. 14-BIO929-03. The authors acknowledge the technical support of Science and Technology Unit, King Abdulaziz University.

\section{References}

[1] K. G. M. Alberti, P. Zimmet, and J. Shaw, "The metabolic syndrome-a new worldwide definition," The Lancet, vol. 366, no. 9491, pp. 1059-1062, 2005.

[2] S. M. Grundy, J. I. Cleeman, S. R. Daniels et al., "Diagnosis and management of the metabolic syndrome," Circulation, vol. 112, no. 17, pp. 2735-2752, 2005.

[3] N. M. Al-Daghri, O. S. Al-Attas, M. S. Alokail et al., "Diabetes mellitus type 2 and other chronic non-communicable diseases in the central region, Saudi Arabia (Riyadh cohort 2): a decade of an epidemic," BMC Medicine, vol. 9, no. 1, p. 76, 2011.

[4] P. T. Katzmarzyk, I. Janssen, R. Ross, T. S. Church, and S. N. Blair, "The importance of waist circumference in the definition of metabolic syndrome: prospective analyses of mortality in men," Diabetes Care, vol. 29, no. 2, pp. 404-409, 2006.

[5] P. Poirier, T. D. Giles, G. A. Bray et al., "Obesity and cardiovascular disease: pathophysiology, evaluation, and effect of weight loss," Circulation, vol. 113, no. 6, pp. 898-918, 2006.

[6] S. Furukawa, T. Fujita, M. Shimabukuro et al., "Increased oxidative stress in obesity and its impact on metabolic syndrome," The Journal of Clinical Investigation, vol. 114, pp. 1752-1761, 2017.

[7] C. M. Alexander, P. B. Landsman, S. M. Teutsch, and S. M. Haffner, "NCEP-defined metabolic syndrome, diabetes, and prevalence of coronary heart disease among NHANES III participants age 50 years and older," Diabetes, vol. 52, no. 5 , pp. 1210-1214, 2003.

[8] S. Adisakwattana, W. Sompong, A. Meeprom, S. Ngamukote, and S. Yibchok-anun, "Cinnamic acid and its derivatives inhibit fructose-mediated protein glycation," International Journal of Molecular Sciences, vol. 13, no. 2, pp. 1778-1789, 2012.

[9] S.-Y. Goh and M. E. Cooper, "The role of advanced glycation end products in progression and complications of diabetes," The Journal of Clinical Endocrinology \& Metabolism, vol. 93, no. 4, pp. 1143-1152, 2008.
[10] P. Beisswenger and D. Ruggiero-Lopez, "Metformin inhibition of glycation processes," Diabetes \& Metabolism, vol. 29, no. 4, pp. 6S95-6S103, 2003.

[11] G. Vistoli, D. De Maddis, A. Cipak, N. Zarkovic, M. Carini, and G. Aldini, "Advanced glycoxidation and lipoxidation end products (AGEs and ALEs): an overview of their mechanisms of formation," Free Radical Research, vol. 47, no. sup1, pp. 3-27, 2013.

[12] N. Ahmed, R. Babaei-Jadidi, S. K. Howell, P. J. Thornalley, and P. J. Beisswenger, "Glycated and oxidized protein degradation products are indicators of fasting and postprandial hyperglycemia in diabetes," Diabetes Care, vol. 28, no. 10, pp. 2465-2471, 2005.

[13] Y. Higashi, K. Noma, M. Yoshizumi, and Y. Kihara, "Endothelial function and oxidative stress in cardiovascular diseases," Circulation Journal, vol. 73, no. 3, pp. 411-418, 2009.

[14] J. L. Gooch and A. C. Sharma, "Targeting the immune system to treat hypertension," Current Opinion in Nephrology and Hypertension, vol. 23, no. 5, pp. 473-479, 2014.

[15] L. Bahia, L. G. Aguiar, N. Villela et al., "Relationship between adipokines, inflammation, and vascular reactivity in lean controls and obese subjects with metabolic syndrome," Clinics, vol. 61, no. 5, pp. 433-440, 2006.

[16] M. Galleano, V. Calabro, P. D. Prince et al., "Flavonoids and metabolic syndrome," Annals of the New York Academy of Sciences, vol. 1259, no. 1, pp. 87-94, 2012.

[17] G. R. Beecher, "Overview of dietary flavonoids: nomenclature, occurrence and intake," The Journal of Nutrition, vol. 133, no. 10, pp. 3248S-3254S, 2003.

[18] R. Anandhi, T. Annadurai, T. S. Anitha et al., "Antihypercholesterolemic and antioxidative effects of an extract of the oyster mushroom, Pleurotus ostreatus, and its major constituent, chrysin, in Triton WR-1339-induced hypercholesterolemic rats," Journal of Physiology and Biochemistry, vol. 69, no. 2, pp. 313-323, 2013.

[19] A. T. Jan, M. R. Kamli, I. Murtaza, J. B. Singh, A. Ali, and Q. M. R. Haq, "Dietary flavonoid quercetin and associated health benefits-an overview," Food Reviews International, vol. 26, no. 3, pp. 302-317, 2010.

[20] A. Murakami, H. Ashida, and J. Terao, "Multitargeted cancer prevention by quercetin," Cancer Letters, vol. 269, no. 2, pp. 315-325, 2008.

[21] S. Jagtap, K. Meganathan, V. Wagh, J. Winkler, J. Hescheler, and A. Sachinidis, "Chemoprotective mechanism of the natural compounds, epigallocatechin-3-O-gallate, quercetin and curcumin against cancer and cardiovascular diseases," Current Medicinal Chemistry, vol. 16, no. 12, pp. 1451-1462, 2009.

[22] Y. Shen, K. D. Croft, J. M. Hodgson et al., "Quercetin and its metabolites improve vessel function by inducing eNOS activity via phosphorylation of AMPK," Biochemical Pharmacology, vol. 84, no. 8, pp. 1036-1044, 2012.

[23] K. V. Anand, R. Anandhi, M. Pakkiyaraj, and P. Geraldine, "Protective effect of chrysin on carbon tetrachloride (CCl4)induced tissue injury in male Wistar rats," Toxicology and Industrial Health, vol. 27, no. 10, pp. 923-933, 2011.

[24] A. Ahad, A. A. Ganai, M. Mujeeb, and W. A. Siddiqui, "Chrysin, an anti-inflammatory molecule, abrogates renal dysfunction in type 2 diabetic rats," Toxicology and Applied Pharmacology, vol. 279, no. 1, pp. 1-7, 2014.

[25] O. A. A. Ahmed, H. M. El-Bassossy, A. S. Azhar, M. M. Tarkhan, and M. M. El-Mas, "Interference with AGEs formation and AGEs-induced vascular injury mediates curcumin vascular protection in metabolic syndrome," Scientific Reports, vol. 15, no. 10, p. 315, 2020. 
[26] H. M. El-Bassossy, A. A. Elberry, and S. A. Ghareib, "Geraniol improves the impaired vascular reactivity in diabetes and metabolic syndrome through calcium channel blocking effect," Journal of Diabetes and Its Complications, vol. 30, no. 6, pp. 1008-1016, 2016.

[27] H. M. El-Bassossy, R. El-Fawal, A. Fahmy, and M. L. Watson, "Arginase inhibition alleviates hypertension in the metabolic syndrome," British Journal of Pharmacology, vol. 169, no. 3, pp. 693-703, 2013.

[28] H. Abdallah, H. El-Bassossy, G. Mohamed, A. El-Halawany, K. Alshali, and Z. Banjar, "Phenolics from garcinia mangostana inhibit advanced glycation endproducts formation: effect on amadori products, cross-linked structures and protein thiols," Molecules, vol. 21, no. 2, p. 251, 2016.

[29] I. Sadowska-Bartosz, S. Galiniak, and G. Bartosz, "Kinetics of glycoxidation of bovine serum albumin by methylglyoxal and glyoxal and its prevention by various compounds," Molecules, vol. 19, no. 4, pp. 4880-4896, 2014.

[30] F. Abderrahim, S. M. Arribas, M. C. Gonzalez, and L. Condezo-Hoyos, "Rapid high-throughput assay to assess scavenging capacity index using DPPH," Food Chemistry, vol. 141, no. 2, pp. 788-794, 2013.

[31] J. Kaur, "A comprehensive review on metabolic syndrome," Cardiology Research and Practice, vol. 2014, Article ID 943162, 21 pages, 2014.

[32] A. Dhar, I. Dhar, K. M. Desai, and L. Wu, "Methylglyoxal scavengers attenuate endothelial dysfunction induced by methylglyoxal and high concentrations of glucose," British Journal of Pharmacology, vol. 161, no. 8, pp. 1843-1856, 2010.

[33] M. Sánchez, M. Galisteo, R. Vera et al., "Quercetin downregulates NADPH oxidase, increases eNOS activity and prevents endothelial dysfunction in spontaneously hypertensive rats," Journal of Hypertension, vol. 24, no. 1, pp. 75-84, 2006.

[34] H. M. El-Bassossy, S. M. Abo-Warda, and A. Fahmy, "Chrysin and luteolin attenuate diabetes-induced impairment in endothelial-dependent relaxation: effect on lipid profile, AGEs and NO generation," Phytotherapy Research, vol. 27, pp. 1678-1684, 2013.

[35] O. Weinreb, S. Mandel, T. Amit, and M. B. H. Youdim, "Neurological mechanisms of green tea polyphenols in Alzheimer' and Parkinson's diseases," The Journal of Nutritional Biochemistry, vol. 15, no. 9, pp. 506-516, 2004.

[36] C.-H. Wu and G.-C. Yen, "Inhibitory effect of naturally occurring flavonoids on the formation of advanced glycation endproducts," Journal of Agricultural and Food Chemistry, vol. 53, no. 8, pp. 3167-3173, 2005.

[37] N. Al-Musayeib, S. Perveen, I. Fatima, M. Nasir, and A. Hussain, "Antioxidant, anti-glycation and anti-inflammatory activities of phenolic constituents from Cordia sinensis," Molecules, vol. 16, no. 12, pp. 10214-10226, 2011.

[38] H. Y. Kim, B. H. Moon, H. J. Lee, and D. H. Choi, "Flavonol glycosides from the leaves of Eucommia ulmoides O. with glycation inhibitory activity," Journal of Ethnopharmacology, vol. 93, no. 2-3, pp. 227-230, 2004.

[39] S. S. Kang, J. Y. Lee, Y. K. Choi, G. S. Kim, and B. H. Han, "Neuroprotective effects of flavones on hydrogen peroxideinduced apoptosis in SH-SY5Y neuroblostoma cells," Bioorganic \& Medicinal Chemistry Letters, vol. 14, no. 9, pp. 2261-2264, 2004.

[40] L. Naso, E. G. Ferrer, L. Lezama, T. Rojo, S. B. Etcheverry, and P. Williams, "Role of oxidative stress in the antitumoral action of a new vanadyl(IV) complex with the flavonoid chrysin in two osteoblast cell lines: relationship with the radical scavenger activity," JBIC Journal of Biological Inorganic Chemistry, vol. 15, no. 6, pp. 889-902, 2010.

[41] H. Matsuda, T. Wang, H. Managi, and M. Yoshikawa, "Structural requirements of flavonoids for inhibition of protein glycation and radical scavenging activities," Bioorganic \& Medicinal Chemistry, vol.11, no. 24, pp. 5317-5323, 2003.

[42] Q. Liu, T.-T. Chen, and H. Cao, "Flavonoids inhibiting glycation of bovine serum albumin: affinity-activity relationship," Chemical Papers, vol. 69, no. 3, pp. 409-415, 2015.

[43] I. C. Villar, R. Vera, M. Galisteo et al., "Endothelial nitric oxide production stimulated by the bioflavonoid chrysin in rat isolated aorta," Planta Medica, vol. 71, no. 9, pp. 829-834, 2005.

[44] J. Duarte, R. Jiménez, I. C. Villar, F. Pérez-Vizcaíno, J. Jiménez, and J. Tamargo, "Vasorelaxant effects of the bioflavonoid chrysin in isolated rat aorta," Planta Medica, vol. 67, no. 6, pp. 567-569, 2001.

[45] M. Brownlee, "Biochemistry and molecular cell biology of diabetic complications," Nature, vol. 414, no. 6865, pp. 813820, 2001.

[46] K. Nowotny, T. Jung, A. Höhn, D. Weber, and T. Grune, "Advanced glycation end products and oxidative stress in type 2 diabetes mellitus," Biomolecules, vol. 5, no. 1, pp. 194-222, 2015. 